\title{
Chemical changes in irradiated polypropylene studied by X-ray photoabsorption and advanced EPR/ENDOR spectroscopies.
}

\author{
I. Caretti ${ }^{*}$ I. Jiménez ${ }^{*}$ and S. Van Doorslaer ${ }^{* *}$ \\ * Instituto de Ciencia de Materiales de Madrid (ICMM-CSIC), Campus de Cantoblanco, \\ 28049 Madrid (Spain) \\ ** University of Antwerp, Department of Physics, Universiteitsplein 1 (Building N), 2610 \\ Wilrijk, Belgium \\ Correspondence to: I. Caretti (E-mail: caretti@icmm.csic.es)
}

\begin{abstract}
Pulsed and Continuous-wave (CW) Electron Paramagnetic resonance (EPR) in combination with X-ray absorption near edge structure (XANES) analysis is used to study the bulk and surface modification of isotactic polypropylene (i-PP) conducted by X-ray irradiation and $\mathrm{O}_{2}$, Ar and $\mathrm{N}_{2}$ plasma treatments. In all cases, a midchain peroxy radical is the major species detected by CW-EPR, with little delocalization onto the i-PP main chain, as revealed by Xband Mims electron nuclear double resonance and hyperfine sublevel correlation. Besides, surface functionalization of plasma treated i-PP is discussed on the light of XANES results with emphasis in the more complex case of $\mathrm{N}_{2}$ plasma modification. The article gives a comprehensive picture of the chemical changes that occur in irradiated i-PP, with implications in sterilization, surface activation, and adhesion enhancement procedures.
\end{abstract}

Keywords: Polypropylene, irradiation, plasma treatment, Electron Paramagnetic Resonance (EPR), X-ray absorption near edge structure (XANES) 


\section{INTRODUCTION}

Polypropylene is one of the most widely used polymers in the world with applications in the packaging, clothing and medical industries. In these areas, it has become standard practice to use ionizing radiation (electron-beam, UV, gamma rays, ...) for sterilization of the plastic or to modify its mechanical and rheological properties. ${ }^{1-9}$ In turn, surface modification for graft functionalization or for increasing wettability and miscibility of polypropylene is regularly achieved by plasma treatments. ${ }^{10-23}$ More recently, irradiation has also shown to significantly improve the recycling procedures used in polypropylene disposal. ${ }^{24}$ Altogether, the chemical changes induced in this way to the polymer result in the modification of its properties or degradation, mainly through cross-linking, chain scission and grafting mechanisms.

Free radicals, both as intermediate and stable paramagnetic species, are readily formed in polymers by irradiation, and have been extensively studied by Continuous Wave (CW) Electron Paramagnetic Resonance (EPR). ${ }^{25,26}$ In the case of polypropylene, CW EPR has shown that mainly alkyl radicals are produced by vacuum irradiation, which convert into peroxy radicals after air exposure or when the irradiation is conducted under oxic conditions. $^{27-29}$ Peroxy radical species play a vital role in the autoxidation mechanism responsible for polypropylene degradation ${ }^{30-33}$ and have also been considered to be effective as motional spin probes in polymers ${ }^{29,34}$. In general, CW EPR has been regularly applied to explore the type, concentration and dynamics of the abovementioned radicals in polypropylene. However, to our knowledge, no investigations have resorted to the use of advanced pulse EPR techniques to get a deeper insight into the nature of radicals and its interactions within this polymer. The power of pulsed electron nuclear double resonance (ENDOR) and hyperfine sublevel correlation (HYSCORE), for instance, has been recently proven for the analysis of electron beam irradiated polyethylene ${ }^{35}$. 
With respect to non-paramagnetic species, chemical changes (unsaturation, carbonylation, etc) in pure polypropylene or polypropylene blends after irradiation or plasma treatments have been successfully studied by numerous characterization techniques ${ }^{9-12,14-21,36-39}$ : X-ray photoelectron and X-ray absorption spectroscopies, Auger electron spectroscopy, infrared spectroscopy, optical absorption, chromatography, atomic force and transmission electron microscopies, etc. Among them, the use of X-ray absorption near edge spectroscopy (XANES) is particularly attractive for surface modified polymers, providing us with element, site and local structure specific information. The presence and orientation of conjugated carbon $\mathrm{C}=\mathrm{C}$ bonds, as well as $\mathrm{C}-\mathrm{O}$ and $\mathrm{C}=\mathrm{O}$ functional groups has been determined by XANES in photodegraded ${ }^{40}$ and oxygen plasma treated ${ }^{13,15,41}$ polypropylene.

Albeit polypropylene irradiation is regularly applied for many technological applications, a complete picture of the chemical species formed is still the subject of active debate, since most studies focus either on the free radical chemistry or the non-paramagnetic chemical species. In this work, we have exploited the combination of CW and pulsed EPR/ENDOR advanced techniques together with XANES in order to explore all the chemical changes induced by irradiation of polypropylene. Specifically, PP was irradiated with X-rays under air conditions and using $\mathrm{N}_{2}, \mathrm{O}_{2}$ and $\mathrm{Ar}$ plasmas inside a low pressure radiofrequency chemical vapour deposition reactor.

\section{EXPERIMENTAL}

Isotactic polypropylene (i-PP) powders and films. The pristine polypropylene was an isotactic homopolymer in pellet form, provided by REPSOL. It is characterized by a polydispersity of 4.77 , an isotacticity of $95 \%$, determined by solution NMR, and a viscosity average molecular weight of $179,000 \mathrm{~g} / \mathrm{mol}$, obtained by intrinsic viscosity measurements. The i-PP raw material was prepared in two geometries: (1) as micrometric powder to enhance 
the surface-volume ratio and allow the detection of surface radicals with EPR, and (2) as thin films to minimize electric charge accumulation in the course of XANES spectroscopy.

i-PP powders were obtained through a solution-precipitation procedure by diluting micrometric pellets in xylene in concentration of $7.5 \mathrm{~g} / 1$ at $120^{\circ} \mathrm{C}$ and subsequent precipitation with methanol at room temperature in 1:4 proportions. The resulting polymers were examined with a Nova NanoSEM 230 FEI Scanning Electron Microscope (SEM) using a landing energy of $1 \mathrm{keV}$ to minimize charging effects. The observed average size of polypropylene particles was $\sim 3-10 \mu \mathrm{m}$.

For the XANES measurements, i-PP was shaped as thin films with thicknesses between 1 and $10 \mu \mathrm{m}$, and were not used as free standing films but as coatings of stainless steel substrates in order to maximize electrical contact to a conductor. To prepare the films, the PP pellets were compression moulded on steel plates at 100 bar by heating the pellets at $210^{\circ} \mathrm{C}$ for 5 min with subsequent quenching of the formed film between water-cooled metal plates.

X-ray irradiation. All polymers were irradiated in air at room temperature with X-rays from a Siemens Kristalloflex X-ray tube equipped with a $\mathrm{Cu}$ anode using $50 \mathrm{~mA}$ current and $50 \mathrm{kV}$ voltage. The samples were placed $7 \mathrm{~cm}$ below the $\mathrm{X}$-ray source.

Plasma treatment. We used a low pressure RF plasma reactor composed of a quartz cylinder of $750 \mathrm{~mm}$ length and $61 \mathrm{~mm}$ inner diameter, evacuated down to $1.10^{-3} \mathrm{mbar}$ with a rotary pump (Leybold-DUO 35), where high purity gases were fed. The RF excitation is provided by a $13.56 \mathrm{MHz}$ generator (Hüttinger-RF300), with impedance adjustment until the reflected power was null, inductively coupled to the plasma with a copper coil around the quartz cylinder. The plasma conditions were: $\mathrm{N}_{2}, \mathrm{O}_{2}$ or Ar as gas, an RF power of $100 \mathrm{~W}$, total pressure in the reactor chamber of $0.1 \mathrm{mbar}\left(\right.$ e.g. $\sim 30 \mathrm{sccm}$ flow rate for $\left.\mathrm{N}_{2}\right)$ and a treatment time of $30 \mathrm{~s}$. Treatment times longer than $30 \mathrm{~s}$ increase significantly the polymer damage by chain scission and cross-linking. 
XANES. X-ray photoabsorption experiments were carried out at the PM4 beamline of the BESSY II synchrotron source in Berlin (Germany) using the SurICat endstation. The spectra were collected in the total electron yield mode by registering the current drained to ground from the sample, which was normalized by the current from a gold-coated grid placed upstream in the x-ray beam path.

Continuous-wave (CW) EPR. X-band ( 9.44GHz) CW-EPR spectra were recorded at 5K, $100 \mathrm{~K}$ and room temperature on a Bruker ESP300 spectrometer equipped with a continuous gas flow cryogenic system (model EPR910 from Oxford Instruments). The X-band EPR experiments were performed using a microwave power of $0.1 \mathrm{~mW}$ (at $5 \mathrm{~K}$ ) and $2 \mathrm{~mW}$ (at $100 \mathrm{~K}$ or higher) in the 328-342 mT magnetic field range with a modulation amplitude of 0.2 mT and a modulation frequency of $100 \mathrm{kHz}$. In turn, W-Band CW-EPR ( 94.0GHz) was performed at $100 \mathrm{~K}$ on a Bruker Elexsys E680 spectrometer equipped with a continuous gas flow cryostat from Oxford Instruments. All W-Band EPR spectra were recorded using $0.011 \mathrm{~mW}$ microwave power, whilst magnetic field was swept in the 3367-3290mT range with a modulated frequency and amplitude of $100 \mathrm{kHz}$ and $0.3 \mathrm{mT}$, respectively. EPR simulations were done with the Matlab Easyspin package developed at ETH Zurich. ${ }^{42}$

X-band pulsed EPR spectroscopy. The X-band pulsed EPR experiments were performed at $10 \mathrm{~K}$ on a Bruker ESP380 spectrometer (mw frequency $~ 9.78 \mathrm{GHz}$ ) equipped with a liquidhelium cryostat from Oxford Inc. All experiments were done at $10 \mathrm{~K}$ with a repetition rate of $123 \mathrm{~Hz}$. ENDOR (electron nuclear double resonance) spectra were obtained with a Odb RF power from an ENI 3100L RF amplifier. The magnetic field was measured with a Bruker ER035M NMR Gaussmeter. The pulsed ENDOR measurements were recorded using the Mims $\operatorname{ENDOR}^{43}$ pulse sequence $\pi / 2-\tau-\pi / 2-T-\pi / 2-\tau$-echo, with $\tau=120 \mathrm{~ns}, t_{\pi / 2}=16 \mathrm{~ns}$, and $\mathrm{T}=17.7 \mu \mathrm{s}$. A $16 \mu$ s rf pulse was applied during time interval $T$ with a variable frequency 
between 5-25 $\mathrm{MHz}$ in steps of $50 \mathrm{kHz}$. HYSCORE (hyperfine selective correlation) experiments ${ }^{44}$ consisted in the pulse sequence $\pi / 2-\tau-\pi / 2-t_{1}-\pi-t_{2}-\pi / 2-\tau$-echo, with pulse lengths of $t_{\pi / 2}=t_{\pi}=16 \mathrm{~ns}$. Times $t_{1}$ and $t_{2}$ were varied from $104 \mathrm{~ns}$ to $3704 \mathrm{~ns}$ in steps of $8 \mathrm{~ns}$ and $\tau=120 \mathrm{~ns}$. Besides, the second and third $t_{\pi / 2}$ pulses were matched to maximize the proton modulation depth. An eight-step phase cycle was used to eliminate unwanted echoes. The individual time traces were baseline corrected with a third-order polynomial, apodized with a Hamming window and zero-filled. After 2D Fourier transformation, the absolute-value spectrum was calculated.

\section{RESULTS}

\section{X-ray irradiation of polypropylene (PP). \\ a. CW EPR.}

The X-band CW EPR of polypropylene was measured before and after ex-situ X-ray irradiation in order to study the formation of free radicals under oxic conditions. CW-EPR spectrum (a) in figure 1 for the non-irradiated polypropylene sample at $100 \mathrm{~K}$ confirms the absence of native paramagnetic species in the polymer powder. Only a small radical signal from the quartz tube is observed (marked with an asterisk). In contrast, after $30 \mathrm{~s}$ X-ray irradiation of polypropylene a clear anisotropic EPR signal is detected, which is typical of a midchain peroxy radical. ${ }^{29}$ Figure 1 shows the normalized spectra (b), (c) and (d) of this radical species recorded at $298 \mathrm{~K}, 100 \mathrm{~K}$ and $5 \mathrm{~K}$, respectively. For decreasing temperature, we observe a slight, but significant increase of $\sim 0.0012$ of the $g_{3}$ value and almost no change of the $\left(g_{1}, g_{2}\right)$ principal $g$ values (see dotted line). The spectrum signal to noise ratio is worse at $5 \mathrm{~K}$ due to a saturation effect. The measured $g$ anisotropy and temperature dependence is well known for peroxy radicals in polypropylene, and has been successfully described by rotation of the radical around the $\mathrm{C}-\mathrm{O}$ bond axis. ${ }^{29,34}$ 


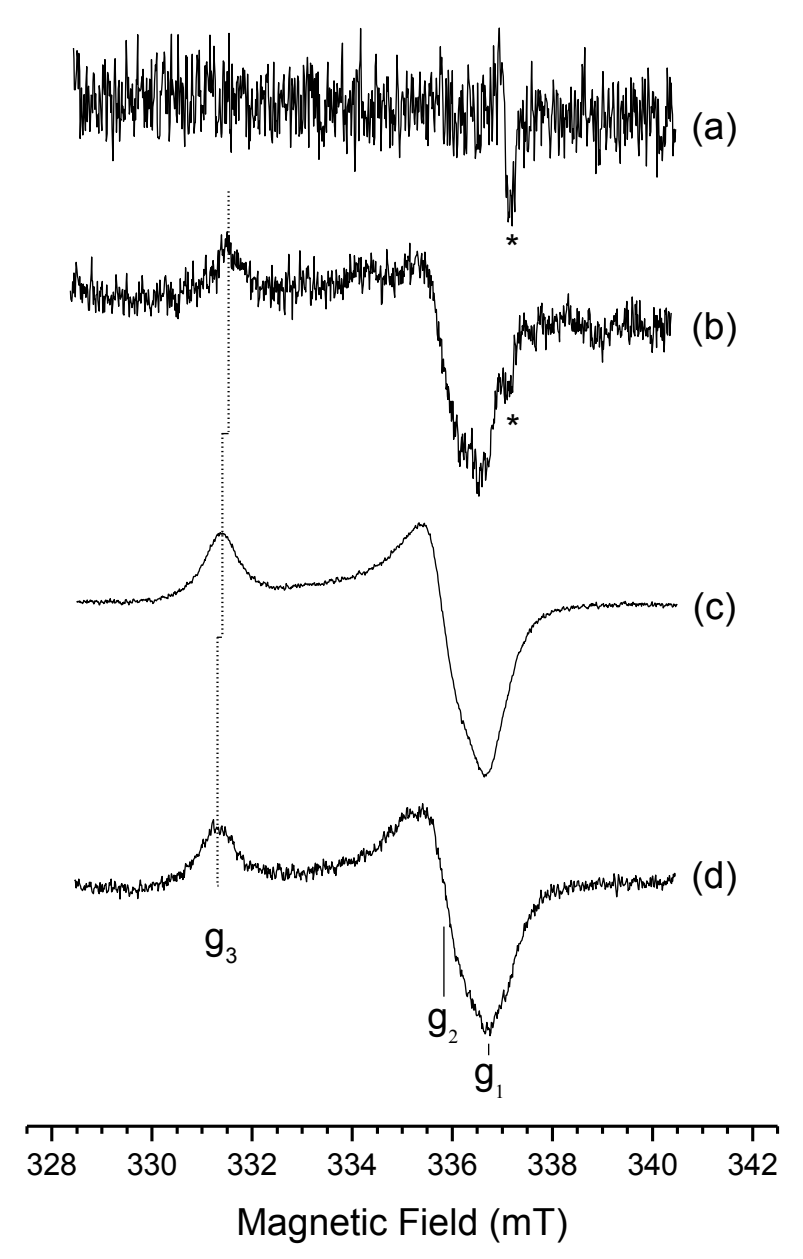

Figure 1. X-band CW-EPR spectra of (a) non-irradiated polypropylene and X-ray irradiated polypropylene at (b) room temperature, (c) $100 \mathrm{~K}$ and (d) $5 \mathrm{~K}$. A temperature dependent increase of $g_{3}$ is indicated by a dotted line and the signal from the empty quartz tube is marked with an asterisk.

The W-band CW-EPR measurements at $100 \mathrm{~K}$ of polypropylene irradiated with X-rays for 30 $\mathrm{s}$ and $120 \mathrm{~s}$ are depicted in figure 2. A manganese signal from the $\mathrm{W}$-band cavity is indicated with asterisks. At $100 \mathrm{~K}$, both $\mathrm{X}$ - and W-band spectra could be simulated with $\boldsymbol{g}=[2.0027$ 2.0085 2.0355], which is characteristic for carbon-based peroxy radicals and consistent with previously reported Q band experiments ${ }^{29,45}$. Interestingly, a new small signal arises at Wband with a slightly higher $g_{3}=2.0375$ (see enlarged insert), which could also be the origin of the tail observed at the low-field side of the X-band spectra.. This indicates a low concentration of a second peroxy radical conformation. 


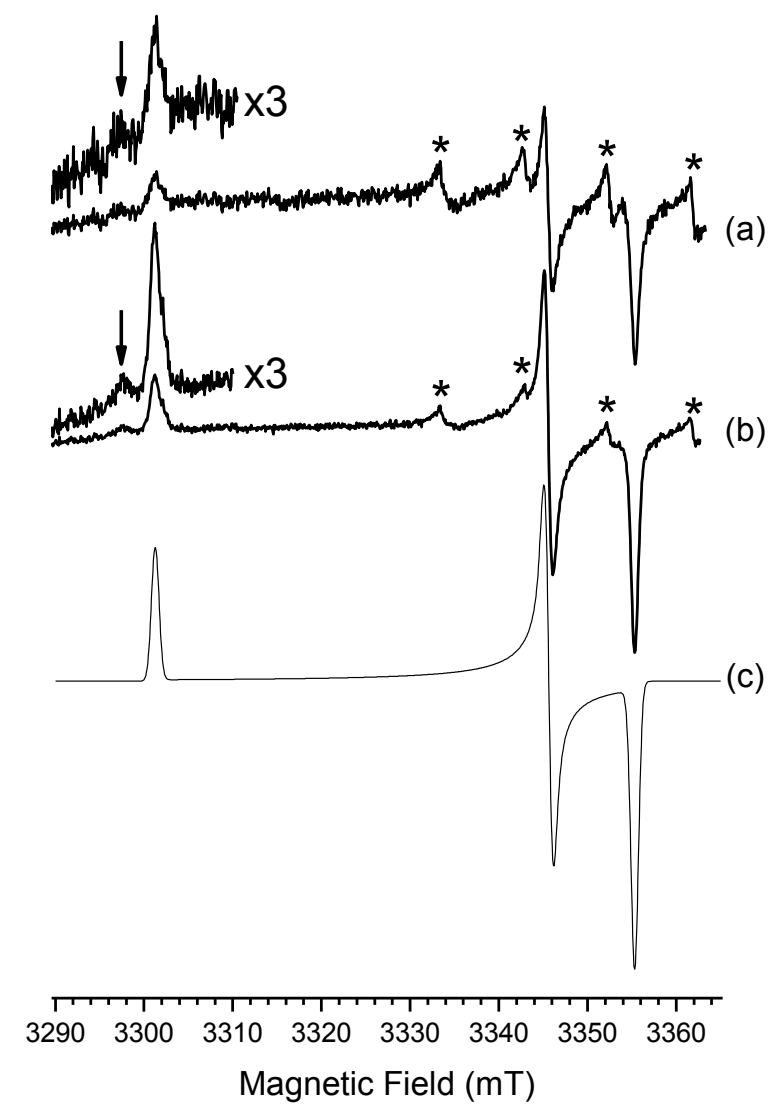

Figure 2. W-band CW-EPR spectra of polypropylene recorded at 100K after X-ray irradiation for (a) $30 \mathrm{~s}$ and (b) $120 \mathrm{~s}$. (c) Simulation of the EPR spectra with $g=[2.00262 .0085$ 2.0355]. Peaks belonging to a Mn signal from the cavity are marked with asterisks.

\section{b. Pulse EPR/ENDOR}

The magnetic field-swept echo experiment of the irradiated polypropylene measured at $10 \mathrm{~K}$ is shown in Fig. 3 together with the X-band ${ }^{1} \mathrm{H}$ Mims ENDOR recorded at three different observer positions (1)-(3). The ENDOR spectra exhibit a constant width, indicating an isotropic $\mathrm{A}_{0}$ hyperfine interaction with one or more weakly coupled protons. A maximum proton hyperfine splitting of $\sim 4.8 \pm 2 \mathrm{MHz}$ is determined, in agreement with the $\mathrm{X}$-band ${ }^{1} \mathrm{H}$ matched HYSCORE spectra measured at position (3) and shown in Fig.4. 
(3)

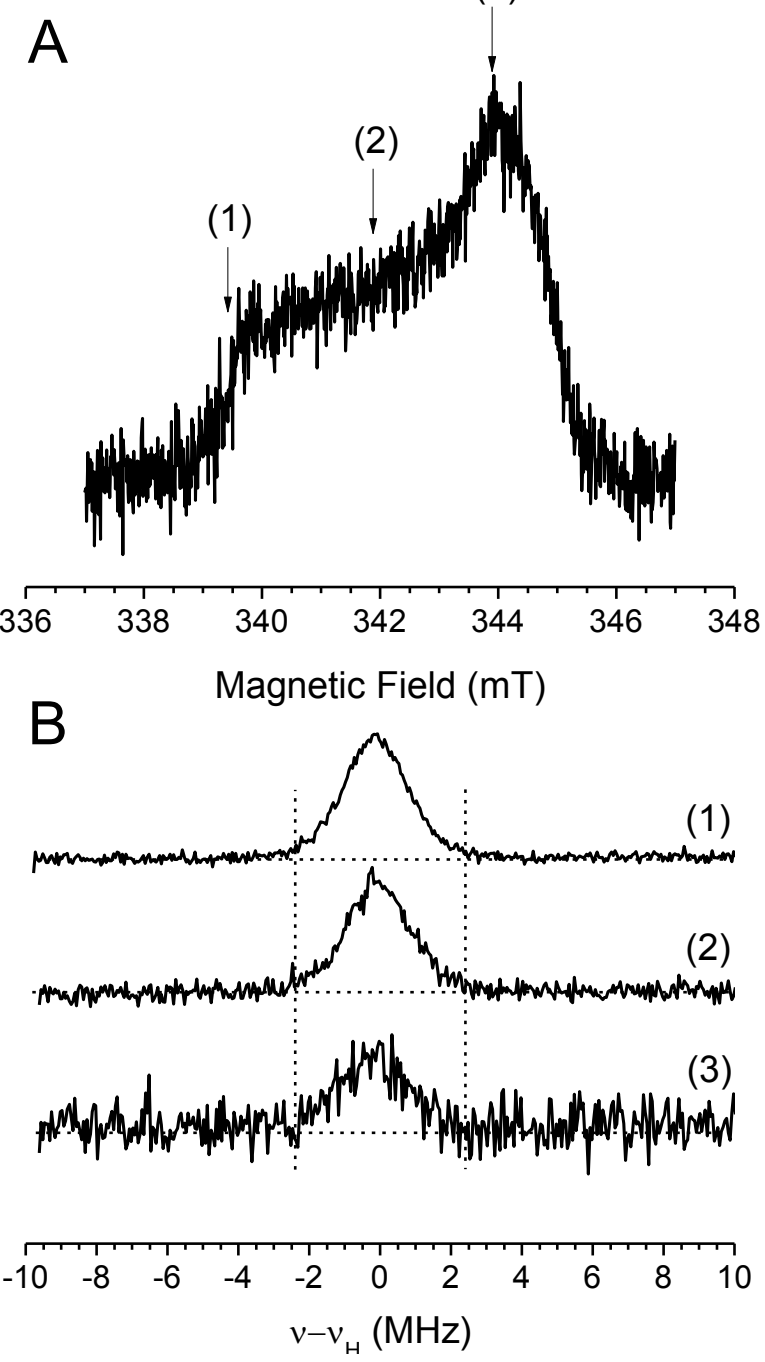

Figure 3. (A) Magnetic field-swept echo intensity and (B) X-band 1H Mims ENDOR spectra recorded at 10K for X-ray irradiated polypropylene at observer positions (1), (2) and (3).

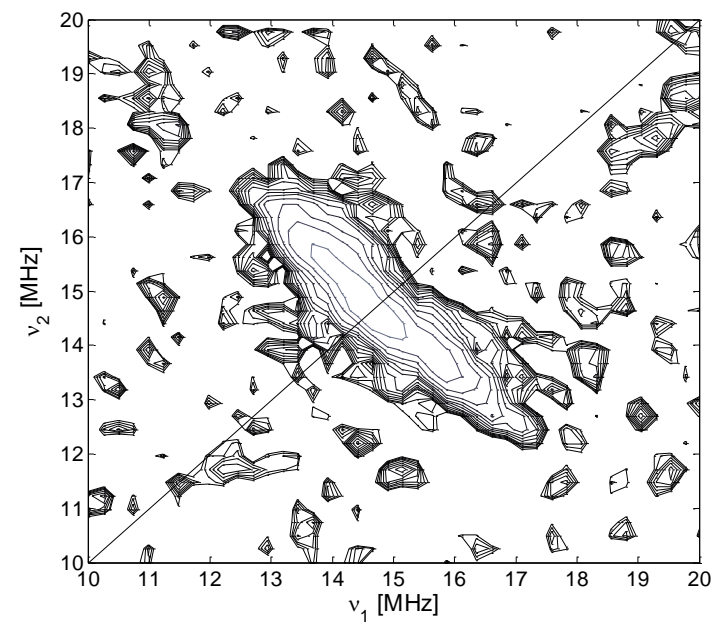

Figure 4. X-band ${ }^{1} \mathrm{H}$ matched HYSCORE spectrum of X-ray irradiated polypropylene recorded at $10 \mathrm{~K}$ at observer position (3) (see figure 3). 


\section{Plasma treatment}

\section{a. CW EPR}

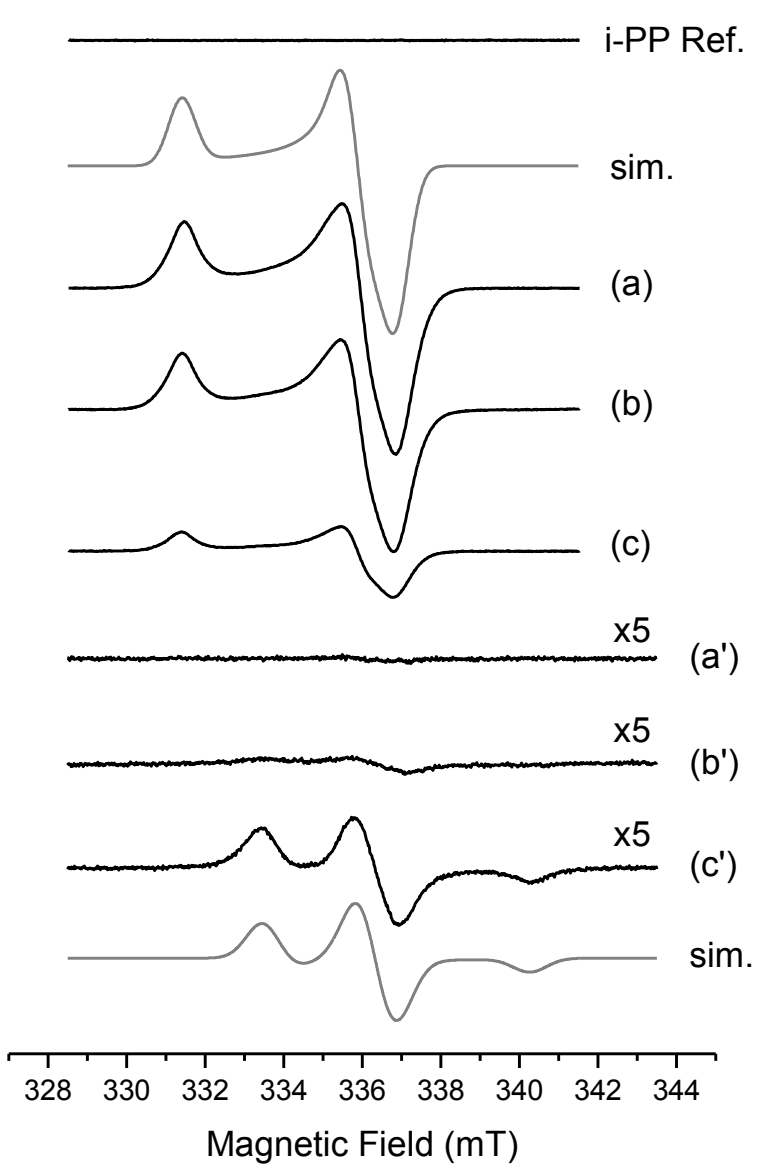

Figure 5. X-band CW-EPR spectra at $5 \mathrm{~K}$ of (a) $\mathrm{O}_{2}$, (b) Ar and (c) $\mathrm{N}_{2}$ plasma modified polypropylene, one week after treatment. (a',b' and c') spectra correspond to the same samples but one year after the plasma treatment.

The X-band CW-EPR spectra of (a,a') $\mathrm{O}_{2}$, (b,b') $\mathrm{Ar}$ and (c,c') $\mathrm{N}_{2}$ plasma modified polypropylene measured at $5 \mathrm{~K}$ are depicted in Fig. 5, corresponding to one week (a,b anc c) and one year ( $a$ ', $\mathrm{b}^{\prime}$ and $\left.\mathrm{c}^{\prime}\right)$ after treatment samples. While the pristine polymer reference displays no EPR signal, the same free radical spectrum is observed for the three different gases used for modification after one week, which is identical to the one detected in X-ray irradiated polypropylene due to midchain peroxy radicals. Simulation of this radical species using the $\boldsymbol{g}$ values obtained from W-band EPR is shown in grey. According to the spectra intensity, an increasing amount of these peroxy radicals is formed with $\mathrm{N}_{2}, \mathrm{Ar}$ and $\mathrm{O}_{2}$ 
plasmas. After one year, the EPR spectra have changed completely. A new signal from a more stable radical is detected in place of the peroxy radical for the $\mathrm{N}_{2}$-modified polypropylene, whereas it is almost absent in the $\mathrm{Ar}$ and $\mathrm{O}_{2}$ modified samples. Hence, we have assigned it to a nitroxide radical with $\boldsymbol{g}=\left[\begin{array}{lll}2.0025 & 2.0066 & 2.0089\end{array}\right]$ and $\boldsymbol{A}=\left[\begin{array}{lll}94.8 & 12.3 & 7\end{array}\right]$ $\mathrm{MHz}$ (see simulated spectra in grey). Notice that these spectra have been magnified by 5 times, indicating a lower concentration compared to the peroxy radicals.

\section{b. XANES}

The survey XANES spectra (280-560eV) of pristine polypropylene and plasma treated with $\mathrm{O}_{2}$, Ar and $\mathrm{N}_{2}$ are depicted in the top panel of Fig 6. They clearly show that for all gases the plasma treatment incorporates oxygen into the polymer structure, while nitrogen is only detected for the $\mathrm{N}_{2}$-modified polypropylene. Below the top panel, a detailed view of the Xray absorption at the $\mathrm{C} \mathrm{K}-, \mathrm{N} \mathrm{K}$ - and $\mathrm{O} \mathrm{K}$-edges is shown. For simplicity, we only discuss here the spectra measured after $\mathrm{N}_{2}$ modification, since similar chemical changes are observed in the polymer at the $\mathrm{C} \mathrm{K}$ - and $\mathrm{O} \mathrm{K}$-edges for the other gases.

The C K-edge XANES of untreated polypropylene consists of two characteristic peaks at $\sim 288$ and $\sim 292.5 \mathrm{eV}$, assigned to $\sigma^{*}(\mathrm{C}-\mathrm{H})$ and $\sigma^{*}(\mathrm{C}-\mathrm{C})$ resonances. $^{41}$ Although, according to its structure, polypropylene should not exhibit $\pi^{*}$ features (all $\mathrm{C}$ atoms are $\mathrm{sp}^{3}$-hybridized), some intensity is detected between $282-286 \mathrm{eV}$ due to the formation of terminal double bonds or surface contaminant species. ${ }^{41}$ After $\mathrm{N}_{2}$ plasma treatment, there is an increase of the $\pi^{*}$ intensity linked to the formation of carbon double bonds and two pre-edge peaks A and B are resolved at $\sim 284$ and $\sim 285.4 \mathrm{eV}$, respectively. Peak A and B have been previously observed in many carbon-based materials and originate from both aromatic and olefinic carbon double bonds. ${ }^{46-49}$ By direct comparison with the pristine polypropylene C K-edge, and based on structural similarities, we interpret the appearance of these bonding environments to the 
generation of alkene linear chains and graphitization in the surface of the polymer as a result of plasma dehydrogenation.

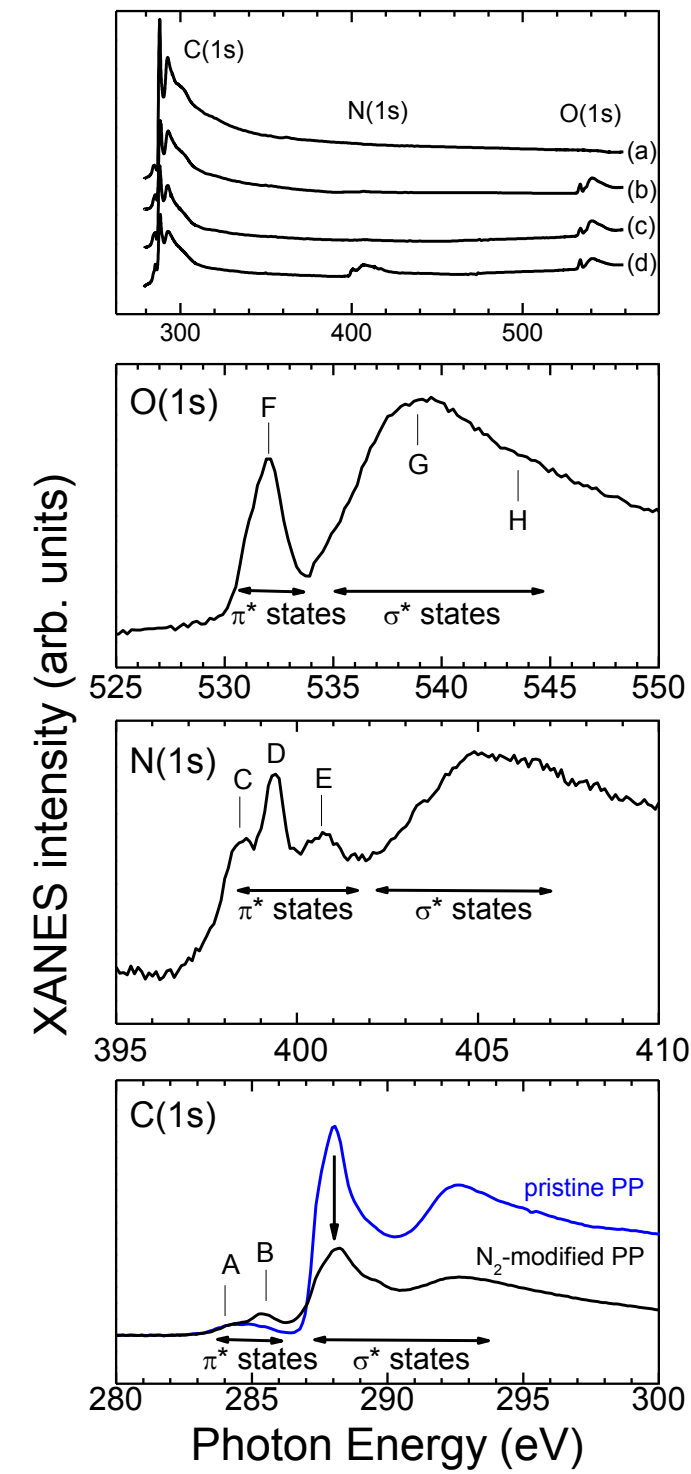

Figure 6. Survey XANES spectra of (a) pristine polypropylene and (b) $\mathrm{O}_{2^{-}}$, (c) Ar- and (d) $\mathrm{N}_{2}$-plasma treated polypropylene (top panel). A detailed view of the absorption at the $\mathrm{O}, \mathrm{C}$ and $\mathrm{N} \mathrm{K}$-edges is shown below for the nitrogen modified polypropylene, together with the $\mathrm{C}$ K-edge of unmodified pristine polypropylene (blue).

In turn, the $\sigma^{*}(\mathrm{C}-\mathrm{H})$ peak characteristic of polyolefins is reduced and broadened due to the incorporation of $\mathrm{N}$ and $\mathrm{O}$ atoms, which produce new unresolved features consistent with chemical changes in the polymer. Their identification is nontrivial, since many distinct resonances may coexist in the spectral region above $287 \mathrm{eV}$ related to different $\mathrm{C}-\mathrm{O}, \mathrm{C}=\mathrm{O}, \mathrm{C}$ $\mathrm{OH}, \mathrm{C}-\mathrm{H}, \mathrm{C} \equiv \mathrm{N}$ functional groups ${ }^{49}$. As a hint of its complexity, a weak shoulder is detected at $\sim 289.5 \mathrm{eV}$ coincident with transitions present in alcohols and other hydroxylated- and 
ether-linked C species, ${ }^{50}$ plus possible contributions from lower carboxyl resonances that cannot be discarded. ${ }^{47,49}$

The absorption spectrum at the $\mathrm{N}$ K-edge resembles those of carbon nitride compounds, with three distinctive $\pi^{*}$ resonances C-E at $398.5,399.4$ and $400.8 \mathrm{eV}$, respectively, which are widely accepted to arise from 1) pyridine-like environments, 2) nitrile terminal groups and 3) a combination of two nearby peaks from embedded molecular nitrogen and $\mathrm{N}$ bond to $3 \mathrm{C}$ atoms. ${ }^{51}$ Thus, except for the intense nitrile peak $\mathrm{D}$, the other $\pi^{*}$ features are related to the formation of nitrogen-containing aromatic rings. This implies that part of the $\pi^{*}$ features at the $\mathrm{C}$ K-edge must arise from $\mathrm{C}=\mathrm{C}$ in these rings, suggesting some graphitization-like process in the polymer.

With regard to the O K-edge XANES spectrum, a strong peak labeled F is observed at 532.0 $\mathrm{eV}$ due to $\pi^{*}$ states from carbonyl $\mathrm{C}=\mathrm{O}$ groups. ${ }^{49,52}$ Peak $\mathrm{G}$ is typical of $\sigma^{*}(\mathrm{C}-\mathrm{O})$ single bonds, $\sim 5 \mathrm{eV}$ below the analogous $\sigma^{*}(\mathrm{C}=\mathrm{O})$ states marked with an $\mathrm{H}^{49,52}$ Thus, both single and double bond oxygen-based functional groups are formed after plasma modification.

\section{DISCUSSION}

Pristine polypropylene exhibits no native free radicals but has a small concentration of alkene groups as chain terminators or from surface contaminant species, in agreement with previous studies. ${ }^{41}$ After both bulk and surface modification of the polymer by X-ray irradiation and $\mathrm{O}_{2^{-}}$, Ar- and $\mathrm{N}_{2}$-plasma treatments the same midchain peroxy radical species is predominantly formed, with equal anisotropic $\boldsymbol{g}$ tensor. High field W-band EPR allowed an accurate determination of the rhombic $g$ values and showed that a lower concentration of a second peroxy radical species with similar $\left(g_{1}, g_{2}\right)$ and larger $g_{3}$ value is present at longer irradiation times. This increase of the $g_{3}$ value may be accounted for a lower mobility of the peroxy radical in a more rigid environment. ${ }^{53}$ For instance, if a small fraction of midchain 
alkyl radical precursors is formed by demethylation $\left(\mathrm{CH}_{2}-\mathrm{CH}^{\cdot} \cdot \mathrm{CH}_{2}\right)$ instead of the usual dehydrogenation observed by EPR, the motion of the resultant peroxy radical would be constrained by the steric hindrance of the lateral methyl groups in the isotactic polypropylene chain. More than one peroxy species with different mobility have been previously described in polypropylene and other polymers ${ }^{53,54}$.

Interestingly, in the case of $\mathrm{N}_{2}$-plasma treated polypropylene, a third radical species is found after 1 year, which was hidden by the stronger peroxide radical signal in the freshly modified samples. This third species was assigned here to a nitroxide radical from simulations. The formation of a nitrogen-based radical during $\mathrm{N}_{2}$-plasma modification reduces the number of peroxy radicals after exposure to air compared to $\mathrm{Ar}$ and $\mathrm{O}_{2}$ treated polypropylene. In agreement with this, spin labelling of irradiated polypropylene with hindered amine light stabilizers and with other amino additives has led to the formation of nitroxide radicals that inhibit the oxidation mechanism of the polymer. ${ }^{55,56}$ Only traces of the same nitroxide radical are present in the $\mathrm{Ar}$ and $\mathrm{O}$ modified samples, what suggest a negligible nitrogen contamination in the CVD reactor. These results indicate that $\mathrm{N}_{2}$-plasma treatment could be used to impede oxidative degradation in sterilization processes.

The interaction of the main peroxide radical with the molecular surrounding was fully characterized using pulsed EPR techniques. To our knowledge this is the first time such a study is performed, revealing only a weak hyperfine interaction with one or more protons. In agreement with related peroxy species,${ }^{57}$ this result indicates little spin delocalization into the polypropylene chain, showing that most of the unpaired electron density is on the peroxy group in a $\pi$-type orbital. This fact has been connected to the higher activity of polypropylene compared to polystyrene in photodegradation reactions. ${ }^{58}$

In addition, it is noteworthy that plasma treatment of polypropylene results in many chemical changes that are not detected by EPR. XANES shows surface chemical changes that have 
been usually investigated by XPS, with the advantage of clearly distinguishing between the associated $\pi$ and $\sigma$ states. The discussed XANES analysis of $\mathrm{N}_{2}$-plasma modified samples reveals clear signs of functionalization of the polymer in the form of alkene, nitrile and carbonyl groups through dehydrogenation. Moreover, there is proof of $\mathrm{N}$-based aromatic ring formation, which may act as cross linking agents between the polymer chains. These evidences demonstrate the importance of using complementary techniques such as EPR and XANES to get a full picture of the structural changes after polymer surface modification.

In general, the incorporation of polar functionalities and generation of peroxy radicals led to a new type of polypropylene-based material that can be used as a polar compatibilizer to be considered as an additive in inmiscible polymer blends and composites. Actually, these modified polypropylene microparticles were melt-processed to succesfully produce polymer films and polymer nanocomposite films with embedded clay nanoparticles. ${ }^{59}$

\section{CONCLUSIONS}

Midchain peroxy radical is the main paramagnetic species detected by EPR after polpypropylene irradiation with $\mathrm{X}$-rays and $\mathrm{O}_{2}$, Ar and $\mathrm{N}_{2}$ plasmas. This peroxy species are characterized by a large anisotropy and very localized spin density at the O-O atoms. A second minor and more rigid peroxy species appears after longer irradiation times. Furthermore, 1 year aging of the samples shows that peroxy radicals have disappeared, while a more stable nitroxide radical is present in the $\mathrm{N}_{2}$ treated polypropylene that reduces the concentration of peroxide species. Incorporation of nitrogen and oxygen into the polymer through dehydrogenation was confirmed by XANES. Mainly, carbonyl and alkene species were identified in all the plasma modified samples, plus nitrile, nitrogen aromatic rings and embedded molecular nitrogen in the $\mathrm{N}_{2}$ treated ones. The rich diversity of chemical changes 
after polypropylene irradiation shows the importance of using several complementary characterization techniques to identify them.

\section{ACKNOWLEDGEMENTS}

We are indebted to A. Vollmer for her help at the Optics beamline (SURICAT endstation) of the Helmholtz-Zentrum Berlin - Electron storage ring BESSY II. The research leading to these results has received funding from the European Community's Seventh Framework Programme (FP7/2007-2013) under grant agreement n.226716. This work has been partially financed by the Spanish MICINN through project Consolider FUNCOAT CSD2008-0023, and project MOCASINN MAT2010-21070-C02-02. I. Caretti acknowledges support from CSIC through a JAE-Doc fellowship. S. Van Doorslaer acknowledges support from the Hercules Foundation, Flanders (contract AUHA013)

\section{REFERENCES}

1 D. Jiri George. Ionizing Radiation and Polymers; William Andrew Publishing, 2013.

2 Y.-C. Tyan; J.-D. Liao; S.-P. Lin; C.-C. Chen. J. Biomed. Mater. Res. A 2003, 67A, 1033-1043.

3 K. A. d. S. Aquino. In Gamma Radiation; Adrovic, F., Ed.; InTech, 2012, chapter 9, pp 171-206.

4 A. C. Abraham; M. A. Czayka; M. R. Fisch. Rad. Phys. Chem. 2010, 79, 83-92.

5 A. E. Goulas; K. A. Riganakos; M. G. Kontominas. Rad. Phys. Chem. 2004, 69, 411417.

6 A. E. Goulas; K. A. Riganakos; M. G. Kontominas. Rad. Phys. Chem. 2003, 68, 865872.

7 M. M. H. Senna; A. A. Abdel-Fattah; Y. K. Abdel-Monem. Nucl. Instrum. Meth. B 2008, 266, 2599-2606.

8 A. T. Fintzou; M. G. Kontominas; A. V. Badeka; M. R. Stahl; K. A. Riganakos. Rad. Phys. Chem. 2007, 76, 1147-1155.

9 D. Auhl; J. Stange; H. Münstedt; B. Krause; D. Voigt; A. Lederer; U. Lappan; K. Lunkwitz. Macromolecules 2004, 37, 9465-9472.

10 V. André; F. Arefi; J. Amouroux; G. Lorang. Surf. Interface Anal. 1990, 16, 241-245.

11 M. Collaud Coen; G. Dietler; S. Kasas; P. Gröning. Appl. Surf. Sci. 1996, 103, 27-34.

12 N.-Y. Cui; N. M. D. Brown. Appl. Surf. Sci. 2002, 189, 31-38.

13 J. F. Friedrich; S. Geng; W. Unger; A. Lippitz; J. Erdmann; H. V. Gorsler; C. Wöll; A. Schertel; K. Bierbaum. Surf. Coat. Tech. 1995, 74-75, Part 2, 664-669.

14 N. Gomathi; S. Neogi. Appl. Surf. Sci. 2009, 255, 7590-7600. 
15 T. Gross; A. Lippitz; W. E. S. Unger; J. F. Friedrich; C. Wöll. Polymer 1994, 35, 55905594.

16 F. J. Guild; M. D. Green; R. Stewart; V. Goodship. J Adhesion 2008, 84, 530-542.

17 O.-J. Kwon; S. Tang; S.-W. Myung; N. Lu; H.-S. Choi. Surf. Coat. Tech. 2005, 192, 110.

18 R. Mahlberg; H. E. M. Niemi; F. S. Denes; R. M. Rowell. Langmuir 1999, 15, 29852992.

19 R. Morent; N. De Geyter; C. Leys; L. Gengembre; E. Payen. Surf. Interface Anal. 2008, 40, 597-600.

20 K. N. Pandiyaraj; V. Selvarajan; R. R. Deshmukh; C. Gao. Appl. Surf. Sci. 2009, 255, 3965-3971.

21 N. Shahidzadeh; M. M. Chehimi; F. Arefi-Khonsari; J. Amouroux; M. Delamar. Plasmas and Polymers 1996, 1, 27-45.

22 P. Slepička; A. Vasina; Z. Kolská; T. Luxbacher; P. Malinský; A. Macková; V. Švorčík. Nucl. Instrum. Meth. B, 268, 2111-2114.

23 L. Song; J. Zhao; H. Yang; J. Jin; X. Li; P. Stagnaro; J. Yin. Appl. Surf. Sci., 258, 425430.

24 L. A. Dimitris S. Achilias, Ioannis A. Koutsidis, Dimitra A. Louka, Nikolaos P. Nianias, Panoraia; I. T. a. G. T. Siafaka. In Material Recycling - Trends and Perspectives; Achilias, D., Ed.; InTech, 2012, chapter 1, pp 4-64.

25 B. Rånby; J. F. Rabek. ESR Spectroscopy in Polymer Research; Springer Berlin Heidelberg, 1977.

26 A. Lund; M. Shiotani; S. Shimada. In Principles and Applications of ESR Spectroscopy; Shimada, S., Ed.; Springer Netherlands, 2011, chapter 7, pp 321-378.

27 S. Shimada; Y. Hori; H. Kashiwabara. Macromolecules 1985, 18, 170-176.

28 S. Shimada; Y. Hori; H. Kashiwabara. Macromolecules 1988, 21, 979-982.

29 D. Suryanarayana; L. Kevan. J. Phys. Chem. 1982, 86, 2042-2046.

30 J. L. Williams; T. S. Dunn; H. Sugg; V. Stannett Becton. Rad. Phys. Chem. 1977, 9, 445-454.

31 J. C. W. Chien; C. R. Boss. J. Polym. Sci. A Polym. Chem. 1967, 5, 1683-1697.

32 J. C. W. Chien; C. R. Boss. J. Polym. Sci. A Polym. Chem. 1967, 5, 3091-3101.

33 D. J. Carlsson; C. J. B. Dobbin; D. M. Wiles. Macromolecules 1985, 18, 1791-1793.

34 L. Kevan; S. Schlick. J. Phys. Chem. 1986, 90, 1998-2007.

35 M. C. Paganini; V. Brunella; M. Chiesa. Magn. Reson. Chem. 2012, 50, 615-619.

36 V. André; F. Arefi; J. Amouroux; Y. De Puydt; P. Bertrand; G. Lorang; M. Delamar. Thin Solid Films 1989, 181, 451-460.

37 J. J. Pireaux; R. de Meulemeester; E. M. Roberfroid; C. Grégoire; M. Chtaïb; Y. Novis; J. Riga; R. Caudano. Nucl. Instrum. Meth. B 1995, 105, 186-191.

38 M. Obadal; R. Čermák; M. Raab; V. Verney; S. Commereuc; F. Fraïsse. Polym. Degrad. Stabil. 2005, 88, 532-539.

39 Z. Chen; W. Huang; P. F. Fang; H. M. Wang; S. J. Wang; J. Xiong; Y. S. Xu. Nucl. Instrum. Meth. B 2008, 266, 117-122.

40 P. H. Zhou; O. Kizilkaya; E. Morikawa. Chem. Phys. Lett. 2008, 465, 241-244.

41 W. E. S. Unger; A. Lippitz; C. Wöll; W. Heckmann. Fresen. J Anal. Chem. 1997, 358, 89-92.

42 S. Stoll; A. Schweiger. J. Magn. Reson. 2006, 178, $42-55$.

43 W. B. Mims. Proc. R. Soc. Lond. A Mat. 1965, 283, 452-\&.

44 P. Höfer; A. Grupp; H. Nebenführ; M. Mehring. Chem. Phys. Lett. 1986, 132, 279-282.

45 S. Schlick; L. Kevan. J. Phys. Chem. 1979, 83, 3424-3429.

46 J. Kikuma; B. P. Tonner. J Electron. Spectrosc. 1996, 82, 53-60. 
47 H. Ade; S. G. Urquhart. In Chemicals Applications of Synchrotron Radiation; Sham, T. K., Ed.; World Scientific Publishing Co. Ltd.: Singapore, 2002, chapter 6, pp 285-355.

48 J. Zhou; X. Zhou; R. Li; X. Sun; Z. Ding; J. Cutler; T.-K. Sham. Chem. Phys. Lett. 2009, 474, 320-324.

49 J. T. Francis; A. P. Hitchcock. J. Phys. Chem. 1992, 96, 6598-6610.

50 D. Solomon; J. Lehmann; J. Kinyangi; B. Liang; K. Heymann; L. Dathe; K. Hanley; S. Wirick; C. Jacobsen. Soil Sci. Soc. Am. J. 2009, 73, 1817-1830.

51 R. Gago; I. Jiménez; J. Neidhardt; B. Abendroth; I. Caretti; L. Hultman; W. Möller. Phys. Rev. B 2005, 71, 125414.

52 D. W. Lee; L. De Los Santos V; J. W. Seo; L. L. Felix; A. Bustamante D; J. M. Cole; C. H. W. Barnes. J. Phys. Chem. B 2010, 114, 5723-5728.

53 M. G. Alonso-Amigo; S. Schlick. Macromolecules 1987, 20, 795-801.

54 M. Kuzuya; S.-i. Kondo; M. Sugito; T. Yamashiro. Macromolecules 1998, 31, 32303234.

55 P. Carloni; L. Greci; A. Mar'in; G. Tosi; A. Faucitano. Polym. Degrad. Stabil. 1994, 45, 415-421.

56 P. Silva; C. Albano; R. Perera. Nucl. Instrum. Meth. B 2007, 265, 300-304.

57 A. L. Attwood; J. L. Edwards; C. C. Rowlands; D. M. Murphy. J. Phys. Chem. A 2003, 107, 1779-1782.

58 A. Shyichuk; D. Melnyk; J. R. White. J. Polym. Sci. A Polym. Chem. 2003, 41, 10701076.

59 Z. Martín; I. Jiménez; M. A. Gómez-Fatou; M. West; A. P. Hitchcock. Macromolecules 2011, 44, 2179-2189. 